Volume (4) No. (2) 2021

Equal and Autonomous Living of Secondary Education Pupils from Socially Vulnerable Groups

Xanthippi Foulidi \& Soultana Chouvarda \& Euaggelia Kentrou \& Ioannis Marmaras \& Evangelos C. Papakitsos 


\title{
Equal and Autonomous Living of Secondary Education Pupils from Socially Vulnerable Groups
}

\author{
Xanthippi Foulidi * \\ University of the Aegean, Rhodes, Greece; foulidi@ rhodes.aegean.gr \\ Soultana Chouvarda \\ University of Thessaly, Karditsa, Greece, tania.chouvarda@gmail.com \\ Euaggelia Kentrou \\ University of West Attika, Athens, Greece, kentrouphysio@yahoo.gr \\ Ioannis Marmaras \\ Thriasio General Hospital, Elefsis, Greece, jmarphysio@yahoo.gr \\ Evangelos C. Papakitsos \\ University of West Attica, Egaleo, Greece, papakitsev@uniwa.gr
}

Presented in 22 September 2020

Accepted in $1^{\text {st }}$ December 2020

* Corresponding author

\begin{abstract}
This paper presents two courses for pupils of special vocational secondary education from socially vulnerable groups, that addresses the issues of various stereotypes and discriminations, especially considering gender and cultural ones. Their methodology of implementation is particularly based on constructivism and teamwork. In addition, the interested educators of Special Education may implement these two courses that are described in sufficient details for this matter. A pilot application of them had been conducted in a vocational school of Special Secondary Education, at the city of Elefsis, Greece.
\end{abstract}

Keywords: intercultural communication, stereotypes, social inclusion, gender equality, multiple discriminations. 


\section{Introduction}

The content of the proposed herein program is related to educational activities aimed at the Equal and Autonomous Living of vulnerable groups of pupils who are subject to multiple discriminations, such as people with disabilities and refugees with disabilities. With the aim of mutual respect and diversity, inclusion in education and anti-discrimination, a set of actions and thematic units was designed, which are implemented by pupils under the guidance of their teachers in order to cooperate, interact, create handcrafted educational material, solve everyday problems, adapt, communicate and complete the following two topics:

- $\quad$ Overcoming stereotypes (see section 3);

- Experiential learning through visual means (see section 4).

These activities have been implemented in a vocational school of Secondary Special Education, at the city of Elefsis, Greece, as a part of an ongoing educational project in the context of social awareness and responsibility.

The purpose of this project is to understand the equal treatment of pupils, who are subject to multiple discriminations (due to disability, nationality, gender, race, religion, etc.). More specifically, the various objectives are classified in the following four classes (A-D):

A. For the pupils,

1. To feel safe, through the reduction of xenophobia and racism, adopted by their classmates, as a reflection of what is happening in society and leads to social exclusion.

2. To be empowered to manage emotions and relationships and to overcome the aforementioned difficulties of adaptation both to the social environment and the school environment, that are detrimental to their smooth school and social integration.

3. To facilitate their access to it, in cases where they do not wish to interrupt their schooling.

4. To cultivate, through a set of organized actions, life skills always adapted to the age group they belong to, as well as to their particularities. 
B. For the teachers:

1. To understand the importance and functioning of an open, democratic and multicultural school.

2. To get in touch with intercultural aspects of communication and to know how it takes place in different cultural environments.

3. To understand the negative dimensions of the phenomenon of self-fulfilling prophecy.

4. To familiarize themselves with the methods and content of differentiated teaching, applied in mixed classes.

5. To know the intercultural characteristics that a teacher must have and the way of expressing them.

6. To contribute to the educational activities they will implement, in the creation and organization of a "School for Everyone".

C. For the pupils' parents:

1. To understand the benefits of integrating all children into the school community.

D. For the local community:

1. To realize the benefits of education for all children in the school community.

2. To address social inequality and exclusion, delinquency, violence, the achievement of social cohesion with a substantial contribution to the overall planning for the schools of the future.

The estimated duration of each topic is a month. The teachers, who will implement this program, have the opportunity to choose which topic to start with and to adapt the material to the age and learning needs of their pupils. In addition, they may connect the program with other courses of the curriculum, like Language, Civil Education, Physical Education, Artistic (Fine Arts), History, Autonomous Living, Product Design, Occupational Therapy, Physiotherapy, Psychology and Social Education. Finally, scheduled visits and cooperation with other organizations are possible, like Art galleries and Museums where intercultural programs are implemented. 


\section{Implementation Methodology}

The following teaching methods are applied, according to Botsas \& Sandravelis (2014):

- Adoption of collaborative (teamwork) teaching methods, in which everyone participates and their participation is recognized (Leverett \& Diefendorf, 1992).

- Utilization of new technologies to enhance the learning of vocabulary (Salend, 1994) and any other means (imitations, gestures, change of tone of voice) to understand the meaning of words.

- Utilization of graphic optical means / concept maps, either for the prior cognitive organization of learning or for the depiction of relationships or for the processing of concepts and vocabulary (Raptis \& Rapti, 2014).

- Utilization of elements of the mother tongue and if possible the use of simple expressions, in order to achieve communication with pupils (Brice \& Roseberry-McKibbin, 2001).

- Encouraging the oral repetition of specific expressions, in order for the pupil to become familiar with the tone, rhythm and expression of the language (Salend \& Salinas, 2003).

- Providing immediate feedback by repeating the pupil's comments in the correct language format.

- Adoption of the differentiation of the teaching / learning process; teachers consider not only what they teach, but whom they teach to (Koutselini, 2008: $40)$.

- Encouraging educational visits and worksheets, project method, field study, action research, dating roles, role-playing, communication, trust games.

- Utilization of appropriate educational material, such as images, sketches, songs, movies, fairy tales, plays, literature and comics.

Dramatization and role play, which achieve the change of stereotypical perceptions of pupils, the cultivation of empathy, and therefore the effective communication and effective cooperation with all members of the school community. Literature and biographies are sources for role play. For crisis resolution, the teachers 
approach pupils through case-studies related to intercultural issues. They seek to cultivate dialogue (Nikolaou, 2011), encouraging children to express their personal experiences, promoting their self-esteem and challenging stereotypes and prejudices.

According to constructivism, the learning objects are specific events, real situations, problems that need to be solved. The teachers cultivate the belief that some of the best thoughts come from the collective efforts of a group. Pupils realize that there are many viewpoints on each issue and therefore can often reconsider their own interpretation, taking into account the interpretations of others. Finally, in relation to the above, it is worth a brief and completely indicative reference to the researchdiscovery method, which brings pupils in contact with the applicable rules of logic, which will be the basis of documentation; a viewpoint of them. It is a method that derives its origin from Aristotelian thought, which confirms or rejects an original hypothesis, through justification, documentation, conclusions and generalization. According to Bruner (1961): "the research method uses analytical (or convergent) thinking, which is coherent and progresses through stages, while the exploratory method uses insightful (or divergent) thinking, which follows short paths by making leaps. Examples: Exploring the content of selected websites and presenting information based on specific criteria, examining historical sources, analyzing and applying linguistic rules, etymology of words, origin of proverbs or opinions".

\section{Equal Coexistence: Overcoming Stereotypes}

The aim is for pupils to understand the concepts: stereotype, gender stereotype, social and biological gender, discrimination, diversity and acceptance of diversity by implementing the following three activities, within a proposed schedule of 2 teaching hours per week, for a month.

\section{1st Activity: "The glasses of diversity".}

The attendants watch the video: "The glasses of diversity" (https://www.youtube.com/watch?v=IaW8e9uNDsM) and express their views on the content of the concepts "diversity" and "otherness". Then, they observe the slides and formulate the content of the concepts "stereotype", "prejudice" and "discrimination" (Fig. 1), "gender stereotype", "gender discrimination" (Fig. 2). 


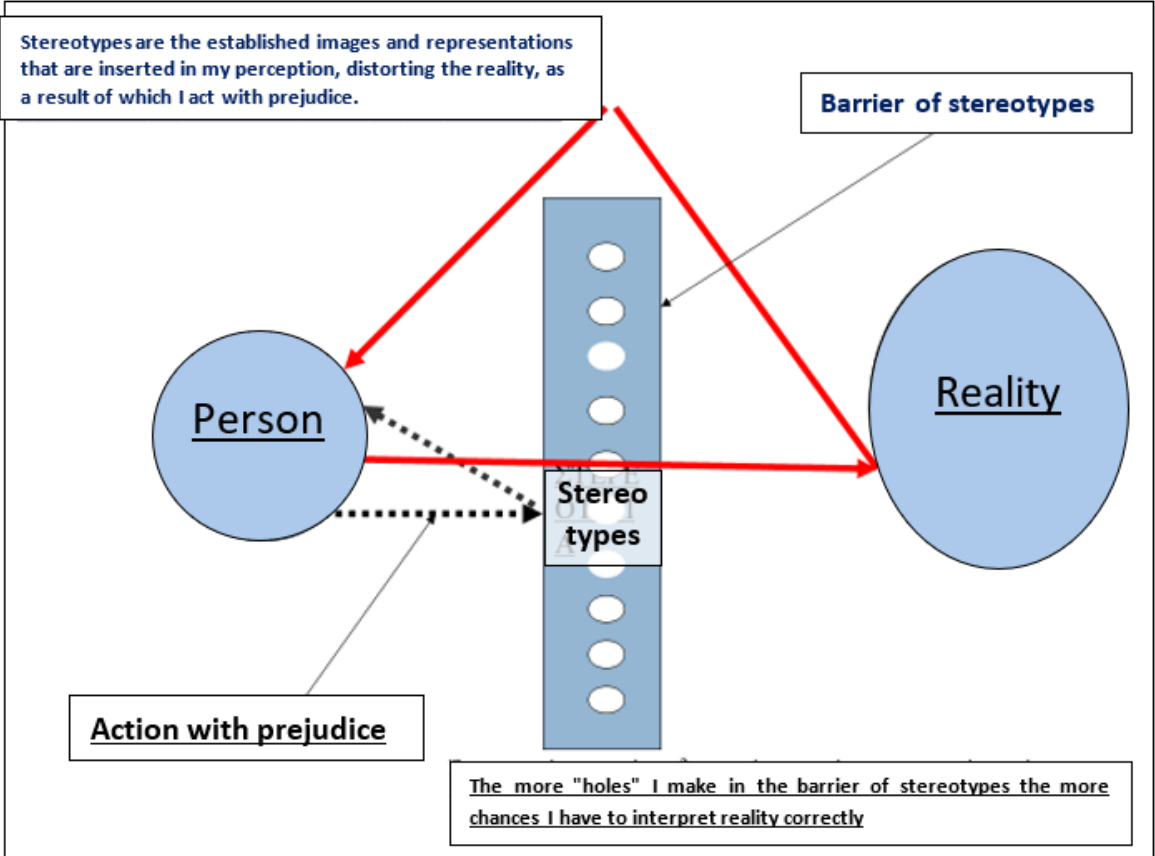

Figure 1. Stereotype, prejudice and discrimination (modified from Foulidi et al., 2020).

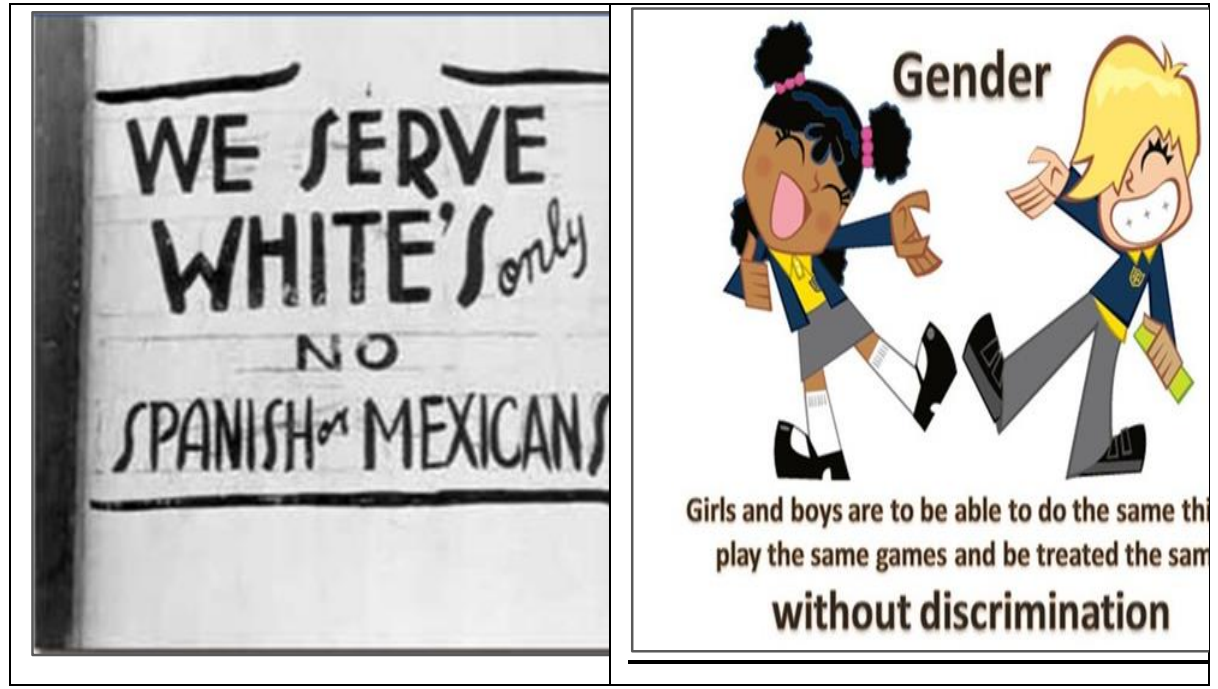

Figure 2. Gender stereotypes and discrimination (in Foulidi et al., 2020). 
Activity 2: Biological and social gender.

Discuss the following phrases (i-xix) with the group of your pupils and ask if they refer to biological or social gender (adaptation from: Promotion of Renewable Energy and Energy Efficiency Programme [PREEEP] (2011), p. 72.; Fig. 3). Discuss gender issues and stereotypes based on the answers.

i. Girls should be gentle and kind, boys should be tough.

ii. Women give birth, men do not.

iii. Dolls are girls' toys.

iv. Boys' voices change during adolescence, while girls' do not.

v. Boys do not Cry.

vi. Men are muscular and physically fit, while women are slender and physically petite.

vii. Men grow beards and mustaches, women do not

viii. Only boys play football.

ix. A girl cannot be a leader in a game.

x. Women have a period, men do not.

xi. Women can breastfeed babies, men cannot.

xii. Women stay at home, and men work for an income.

xiii. Girls are weak and boys are strong.

xiv. Girls help their mothers and boys their fathers.

xv. Girls are smarter than boys.

xvi. Girls win games because they can and do play insidiously.

xvii. It is okay for boys to hit each other but not for girls.

xviii. Boys are lazier than girls.

xix. Girls are better at lying than boys. 


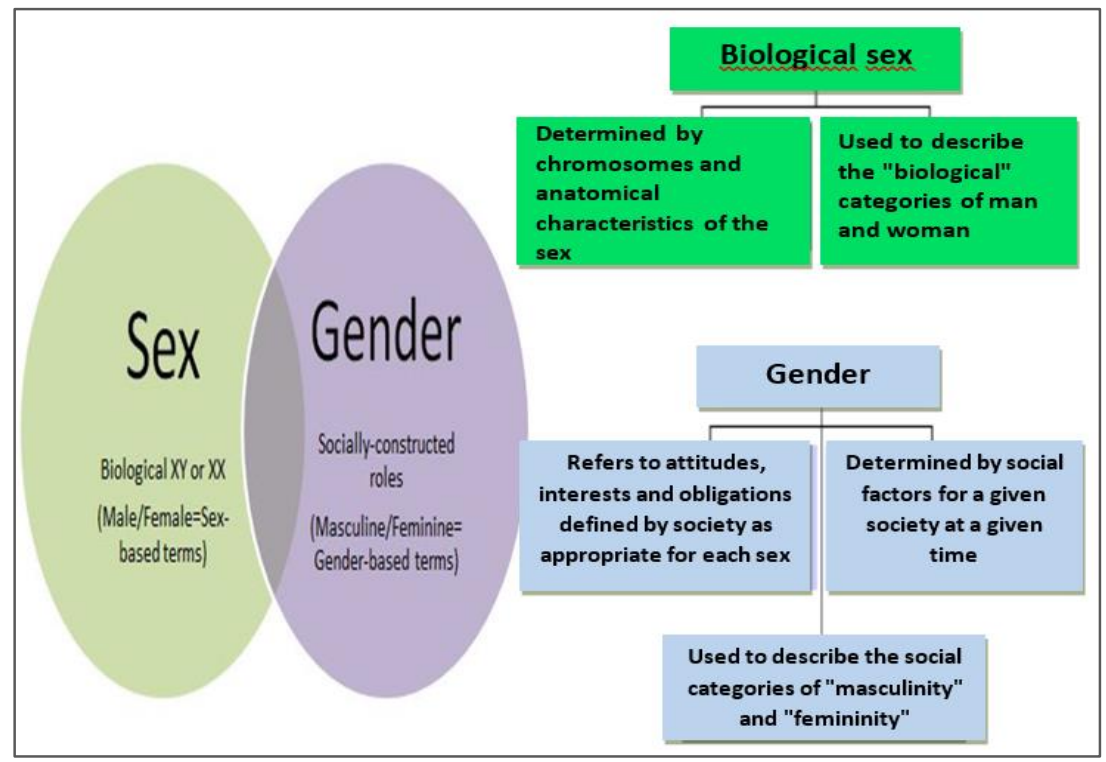

Figure 3. Biological sex and Gender.

Activity 3: Creating a relevant poster.

Pupils create a poster, with the dominant conclusion, which they came to (Fig. 4).

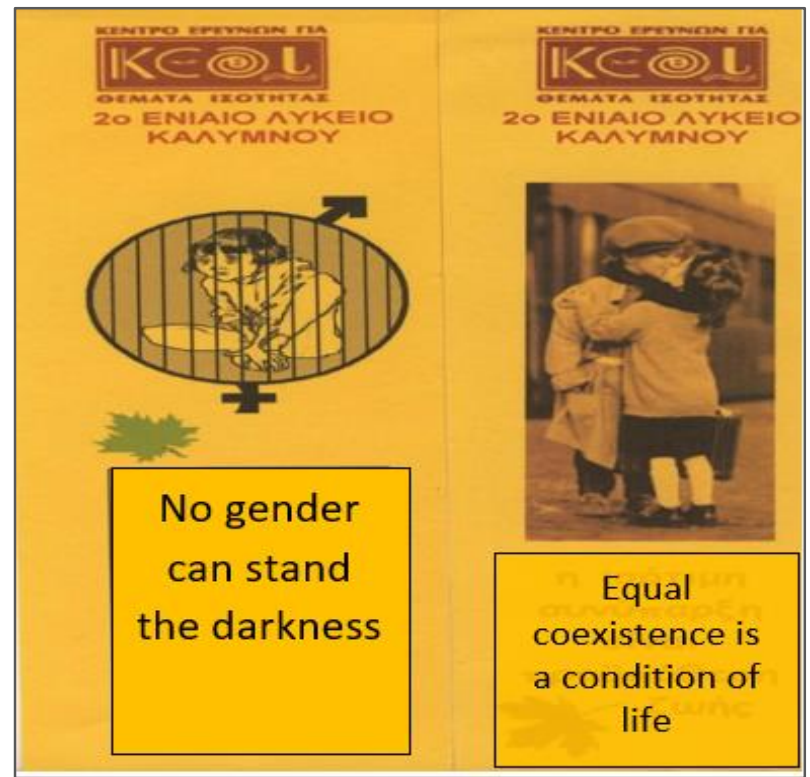

Figure 4. A poster from the $2^{\text {nd }}$ Lyceum of Kalymnos (modified). 


\section{Mutual Respect and Diversity: Implementing Artistic Interventions}

This topic aims to make pupils understand that cultures are equal and the interactions between them are positive. Specific purposes are:

- Understanding the concepts of diversity and equality in visual creations.

- Acquiring skills, such as choosing the appropriate colors, materials, decorative objects depending on the use, the properties and aesthetic effect of each construction to use in artistic activities, which highlight ideas, feelings, objects and their properties; in particular, they promote respect for diversity and parity with their creations, enhancing their visual-motion coordination, their coarse and fine mobility.

- Adopting attitudes, such as empathy, mutual respect, mutual assistance, in order to adopt them in their daily life.

- Collaborating in mixed groups, communicate effectively, take initiatives, adapt, produce, organize and leverage their knowledge of colors, materials and handling to express universal values.

- Developing their critical thinking, responsibility, empathy, creativity.

Teachers should have prepared the space and materials for teaching appropriately, so that:

- To have educational material, such as photos from the internet with themes of people from different parts of the world, in different colors, local costumes, etc.

- Availability of the required materials: paper sheets $\mathrm{A} 4$ size, $\mathrm{PC}$ and projector, pencils, erasers, paints (crayons, markers, acrylics, etc.), cardboard from boxes, scissors, glues, wooden pieces, fabrics, silicone gun, ribbons, twine.

- Be able to sit in a circle.

This topic, scheduled for 1-2 teaching hours per week, consists of the following four stages:

Stage 1: Start of activity. 
The activity begins with a reminder by teachers of the principles of respect for diversity and the value of empathy. Pupils watch a video in which children say the word "good morning" in different languages (https://youtu.be/RoQtvVMTcbI) and listen to songs on the theme of unity and solidarity, such as: "If all the children of the Earth held hands firmly" (https://youtu.be/1Roz0LPjIg4).

Stage 2: Get to know each other Game.

At this initial stage the pupils sit in a $\Pi$ arrangement, at their desks, so that everyone has visual contact with each other. They are asked to write-draw their name, place of origin and next to present a favorite object, hobby, fruit, etc. After completing their work, they are invited to present it to the others. Each time a pupil's presentation is completed, they all applaud and thank each other. Thus, through an artistic activity, they have the opportunity to present themselves and get to know the others. They all have a common goal, exactly the same materials and are free to develop their artistic point of view, but also to get to know each other.

Stage 3: Educational visit to a museum - participation in an artistic activity on the subject of interculturalism.

This activity aims at the artistic coexistence of people with different cultures, backgrounds, disabilities, between pupils who met in the previous stage, to meet again in a museum and participate in an educational program, where they can get to know the Greek and other cultures, through history, folklore, painting, sculpture, games, customs and traditions of antiquity and various cultures. During the implementation of these activities, which take place in various museums (archaeological, cultural and others), it is pointed out that all cultures are equal and the interactions between cultures have positive results. In case of inability to move, due to emergency conditions, a virtual tour can be done via internet, after consultation with the museum of choice.

Stage 4: Collage making, crafts, painting with the theme: A different face.

At this stage, pupils and teachers are invited to participate and jointly design Different Faces (Fig. 5). They can choose to put whatever features they want on the people they will design, e.g., big eyes, different colors, accessories etc. They can collaborate, undertaking to build a face for every three pupils, to submit their ideas, to 
share responsibilities for who will cut the nose, ears, etc., and who will paint or glue the pieces of the face. Through the implementation of this craft, pupils have the opportunity to experience diversity experientially and artistically, to create it, to use imagination, observation, fine and gross mobility, fine motion coordination, initiative, collaboration and above all to experience being equal and free to express their artistic view on the issue of "Different". Different ideas, perceptions, choices for colors, designs, sizes, but with the common goal of creating a common work of a mask face that will include all the different features.
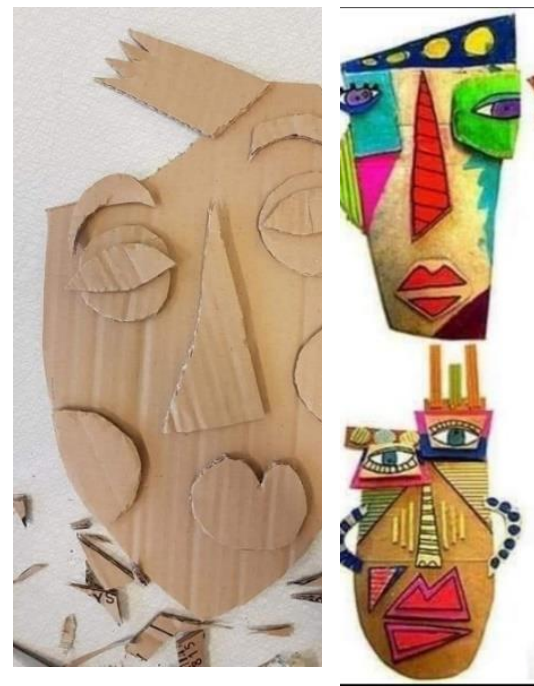
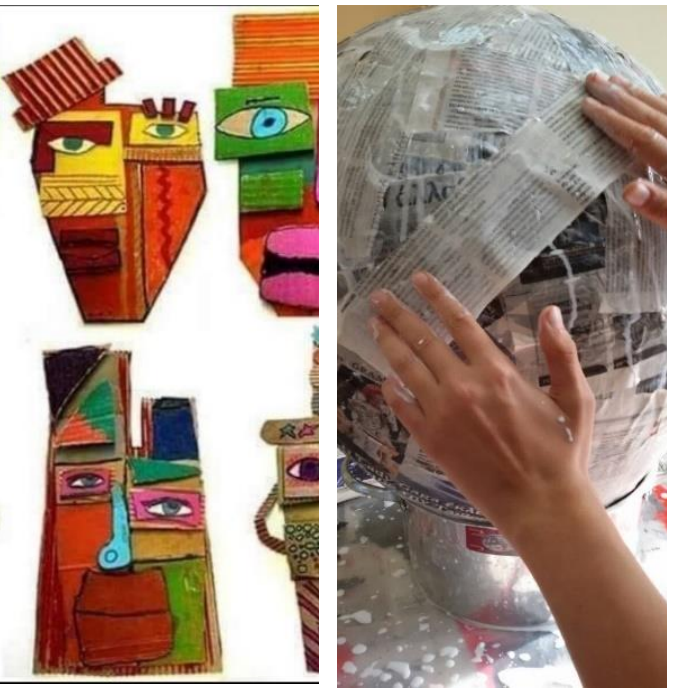

Figure 5. Different Faces.

\section{Conclusions}

The above presented artistic activities can be adapted, depending on the available consumables and supervision materials, the intercultural programs of the museums, the number of participants, the way of their transfer to and from the places of action, the time of implementation, as well as the age and knowledge/level of children. They consider and defend the particularities, the knowledge, and the cultural, religious and educational background of the pupils. The ultimate goal is the acceptance of diversity, through the activities developed and the opportunity for equal participation of all, regardless of their particularities. 
The teachers who will implement this program can choose themselves whether they will carry out the proposed activities or workshops, or they will follow all the educational proposals. Experientiality through learning and inclusion can only have positive effects in the context of educating people with special educational needs and intercultural characteristics. 


\section{References}

Botsas, G., \& Sandravelis, A. (2014). Foreign pupils with special educational needs: the case of special learning difficulties. In E. Katsarou \& M. Liakopoulou (eds.), Issues of teaching and education in the multicultural school Issues of teaching and education in the multicultural school: Educational material. NSRF Program "Education of Foreigners and Repatriated Pupils". Thessaloniki: Greek Ministry of Education and Religious Affairs (in Greek).

Brice, A., \& Roseberry-McKibbin, C. (2001). Choice of languages in instruction: one language or two? Teaching Exceptional Children, 33(4), 10-16.

Bruner, J. S. (1961). The act of discovery. Harvard Educational Review, 31, 21-32.

Foulidi, X., Chouvarda, S., \& Kentrou, E. (2020). Equal and Autonomous Living of Secondary Education Students. Technical Report, Special Vocational Education Laboratory of Elefsis, Greece, (in Greek).

Koutselini, M. (2008). Teaching Construction and Differentiation Learning in mixed ability classes. Nicosia: Self-publishing (in Greek).

Leverett, R. G., \& Diefendorf, A. O. (1992). Pupils with language deficiencies. Teaching Exceptional Children, 24(4), 30-35.

Nikolaou, G. (2011). Intercultural Didactics. Athens: Pedio (in Greek).

Promotion of Renewable Energy and Energy Efficiency Programme (2011). Training Manual on Gender Mainstreaming. Kampala, Uganda: PREEEP.

Raptis, A., \& Rapti, A. (2014). Learning and Teaching in the Information Age. Overall approach. Athens: Self-published (in Greek).

Salend, S. J. (1994). Effective mainstreaming: Creating inclusive classrooms. New York, NY: Macmillan Publishing Co.

Salend, S. J., \& Salinas, A. (2003). Language differences of learning difficulties: the work of the multidisciplinary team. Teaching Exceptional Children, 35(4), 36-43. 